The sustainability of mean-variance and mean-tracking error efficient portfolios

Kris Boudt, Jonathan Cornelissen and Christophe Croux

DEPARTMENT OF DECISION SCIENCES AND INFORMATION MANAGEMENT (KBI) 


\title{
The sustainability of mean-variance and mean-tracking error efficient portfolios
}

\author{
Kris Boudt $^{* \dagger} \quad$ Jonathan Cornelissen $^{\dagger} \quad$ Christophe Croux $^{\dagger}$
}

April 18, 2012

\begin{abstract}
In recent years, the market share of socially responsible investment funds has rapidly increased. This has sparked interest of academics and practitioners for the impact of single stock sustainability screening on portfolio performance. Besides eliminating non sustainable assets from the investment universe, a socially responsible investor also cares about the average level of portfolio sustainability. We provide a characterization of the sustainability of mean-variance and mean-tracking error efficient portfolios and derive the impact on performance of imposing a constraint on the portfolio sustainability. Over the period 2003-2010, we find that for the universe of US stocks belonging to the MSCI World index, the theoretically linear relationship between the portfolio return and sustainability of the efficient portfolios is not significant. Furthermore, we estimate the increase in variance (for a given target return) and the loss in return (for a given target variance) due to a sustainability constraint and find that the performance loss is economically small.
\end{abstract}

Keywords: Mean-variance optimization, Minimum tracking error, Portfolio optimization, Socially responsible investment, Sustainability.

JEL classification: G11, G14, G20.

${ }^{*}$ KU Leuven/Lessius and VU University Amsterdam.

${ }^{\dagger}$ Faculty of Business and Economics, KU Leuven Belgium. E-mail: kris.boudt@econ.kuleuven.be, jonathan.cornelissen@econ.kuleuven.be and christophe.croux@econ.kuleuven.be. 


\section{Introduction}

In January 2010, about $12 \%$ of all professionally managed assets in the United States were invested under the label of sustainable (or socially responsible) investment (Social Investment Forum, 2010). The importance of sustainable investment, which takes into account environmental, social, governance and/or ethical concerns, has fueled an active debate on the impact of the sustainability objective on the investment performance. Previous research has mainly focused on the impact of excluding non-sustainable stocks. Little is known about the performance impact for a socially responsible investor who imposes a target on the portfolio sustainability, defined as the position weighted sustainability of the stocks in the portfolio. We fill this gap by providing a characterization of the sustainability of a mean-variance and mean-tracking error efficient portfolio and by estimating the impact of sustainability restrictions on the portfolio performance.

Regarding the relative performance of sustainable and conventional mutual funds, the consensus of this growing literature seems to be that, after controlling for investment style, the differences in performance tend to disappear (see e.g. Amenc and Le Sourd (2008); Bauer et al. (2005); Derwall and Koedijk (2009) and Statman and Gkushkov (2009)). A second, more recent strand of research has investigated the effect on portfolio performance of excluding stocks based on their sustainability rating. Using the mean-variance spanning test, Galema et al. (2008) and Herzel et al. (2011) find that the economic loss for the investor is small, especially in the presence of no short sales constraints. Screening stocks based on their sustainability rating reflects only one

part of the values-driven sustainable investment practice (as opposed to the group of profit-seeking social investors in Derwall et al. 2011).

In addition to excluding the completely non sustainable assets, the value-driven socially responsible investors also care about achieving a sufficiently high average level of portfolio sustainability (Hallerbach et al., 2004). We are among the first to charac- 
terize the average sustainability level of efficient portfolios and to estimate the effect on portfolio performance of imposing a minimum sustainability condition at the portfolio level. We do this for the frontier of Mean-Variance (MV) and Mean-Tracking error (MTE) efficient portfolios, invested in US stocks belonging to the MSCI World index for the period 2003-2010. While MV efficient portfolios minimize variance subject to a return target, the goal of MTE efficient portfolio optimization is to track a benchmark as closely as possible, while realizing an excess return on top of the benchmark (Roll, 1992). The only related work we know of is an unpublished paper of Drut (2010) who shows that for MV efficient portfolios, the portfolio return and portfolio sustainability are linearly related. As noted by Goltz (2009), the MV efficient portfolio is not so often used in practice, in contrast with the MTE efficient portfolio. The popularity of MTE efficient portfolios stems from the fact that the performance of portfolio managers is typically evaluated with respect to a benchmark. Interestingly, we find that the relation between the portfolio sustainability and the portfolio return is also linear for the MTE efficient portfolio, and moreover, that the slope of this linear relation is the same as for MV efficient portfolios. For the US stocks belonging to the MSCI World index for the period 2003-2010 however, we find that the slope of this linear relation is almost never significantly different from zero.

As a second contribution, we estimate the cost in terms of expected return and variance, caused by imposing a minimum level of portfolio sustainability target. These estimates are important, since they guide the investor in better trading off his objectives of high return, low variance/tracking error variance and a high portfolio sustainability.

The remainder of the paper is organized as follows. In Section 2 we derive the theoretical relationship between portfolio return, variance (tracking error) and sustainability for MV (and MTE) efficient portfolios. Sections 3 and 4 introduce the data and estimation procedure. The results of the sustainability analysis of the MV and MTE 
efficient portfolios of US stocks belonging to the MSCI World over the period 2003-2010 are presented in Section 5. Section 6 concludes the paper and sketches directions for further research.

\section{Sustainability of MV and MTE efficient portfo- lios}

Throughout the paper we study the properties of a portfolio that is fully invested in a universe of $N$ stocks, whereby the investor evaluates the portfolio performance in terms of its expected return, variance (or tracking error) and sustainability. For the latter, the investor relies on a data set of firm specific sustainability scores, stacked in the $N \times 1$ vector $\phi$. The sustainability ratings are standardized in such a way that, for a portfolio with weights $w, w^{\prime} \phi$ measures the portfolio sustainability.

Next, we first review the optimization problem faced by a MV and MTE efficient investor respectively. We then show that for both the MV and MTE efficient portfolios a linear relation exists between portfolio return and portfolio sustainability. Finally, we discuss the solutions to the MV and MTE optimization problem in the presence of a constraint on portfolio sustainability.

\subsection{Review on MV and MTE efficient portfolios}

Denote $\mu$ the $(N \times 1)$ vector with the expected returns and $\Sigma$ their $(N \times N)$ covariance matrix. For a portfolio with weights $\omega=\left(\omega_{1}, \ldots, \omega_{N}\right)^{\prime}$, the portfolio mean and variance are then $\omega^{\prime} \mu$ and $\omega^{\prime} \Sigma \omega$ respectively. These are the key performance statistics for a mean-variance investor, who maximizes the expected portfolio return over the horizon minus the portfolio variance multiplied by the risk aversion parameter $\lambda$, subject to a 
full investment constraint:

$$
\begin{aligned}
& \max _{\omega} \omega^{\prime} \mu-\frac{\lambda}{2} \omega^{\prime} \Sigma \omega, \\
& \text { subject to: } \omega^{\prime} \iota=1,
\end{aligned}
$$

with $\iota$ the vector of ones.

While MV efficient portfolios minimize variance subject to a return target, the goal of MTE efficient portfolio optimization is to track a benchmark as closely as possible, while realizing an excess return on top of the benchmark. The tracking error metric we use is the conventional sum of squared deviations of portfolio returns from the benchmark return, i.e. the tracking error volatility (see e.g. Roll (1992) or Jorion (2003)). The extension to other metrics such as those based on absolute deviations instead of squared deviations (see e.g. Satchell and Hwang (2001) or Rudolf et al. (1999)) is left for future research.

Denote $\tilde{\mu}$ the $(1+N) \times 1$ expected return vector and $\tilde{\Sigma}$ the $(1+N) \times(1+N)$ covariance matrix of the benchmark return and the $N$ assets in the universe jointly. A MTE investor then solves the problem:

$$
\max _{\tilde{\omega}} \tilde{\omega}^{\prime} \tilde{\mu}-\frac{\lambda}{2} \tilde{\omega}^{\prime} \tilde{\Sigma} \tilde{\omega}
$$

subject to: $\tilde{\omega}^{\prime} \iota=0, \tilde{\omega}^{\prime} j=-1$,

with $\tilde{\omega}=(-1 \omega)^{\prime}, j$ the vector $(1,0, \ldots, 0)^{\prime}$ and $\omega$ the vector of weights allocated to the $N$ assets. The constraints in (2.2) imply that $\omega^{\prime} \iota=1$, so $\omega$ represents the weights of the portfolio that tries to mimic the benchmark.

We are interested in the effect on the MV and MTE portfolio performance of con- 
straints on the portfolio sustainability. The portfolio sustainability is given by

$$
\phi_{p}=\omega^{\prime} \phi .
$$

To study this, we first need the portfolio solution in the unconstrained case.

For a risk aversion level $\lambda$, the solution to the MV optimization problem (2.1) is

$$
\begin{gathered}
\omega_{\mathrm{MV}}(\lambda)=a_{\mathrm{MV}}+\frac{1}{\lambda} b, \\
\text { with } a_{\mathrm{MV}}=\frac{\Sigma^{-1} \iota}{\iota^{\prime} \Sigma^{-1} \iota} \text { and } b=\Sigma^{-1}\left(\mu-\iota \frac{\iota^{\prime} \Sigma^{-1} \mu}{\iota^{\prime} \Sigma^{-1} \iota}\right)
\end{gathered}
$$

(see e.g. Best and Grauer (1990)). The optimal MV weights are given by a weighted sum of two weights vectors, where the vector $a_{\mathrm{MV}}$ is weighted by 1 and the vector $b$ by $1 / \lambda$. The weight vector $a_{\mathrm{Mv}}$ corresponds to the minimum variance portfolio, i.e. the portfolio with the lowest variance possible. The minimum variance portfolio is selected by investors having the highest possible risk aversion level $\lambda=+\infty$. Note that the weights in this first weight vector sum to one. Consequently, the weight vector $b$ corresponds to a zero investment portfolio, for which the weights sum to zero. The weights of the zero investment portfolio can be interpreted as the difference in weights with the minimum variance portfolio, needed to obtain a higher return. The higher the risk aversion level $\lambda$ is, the less weight is put on the zero investment portfolio $b$ and the lower the portfolio return becomes.

The return and the variance of the efficient MV portfolio are given as a function of $\lambda$ by $\mu_{\mathrm{MV}}(\lambda)=\omega_{\mathrm{MV}}^{\prime}(\lambda) \mu$ and $\sigma_{\mathrm{MV}}^{2}(\lambda)=\omega_{\mathrm{MV}}^{\prime}(\lambda) \sum \omega_{\mathrm{MV}}(\lambda)$ respectively. For $\lambda \geq 0$, the solutions $\left(\mu_{\mathrm{MV}}(\lambda), \sigma_{\mathrm{MV}}^{2}(\lambda)\right)$ give rise to a hyperbola in the portfolio mean versus portfolio variance plane. This hyperbola is typically referred to as the MV efficient frontier. The solid line in the left panel of Figure 1 plots the MV efficient frontier for illustrative purposes. 
Figure 1: MV and MTE efficient frontier (left) and daily portfolio return in percentage points as a function of portfolio sustainability for the portfolios on the efficient frontier (right) for a universe of US stocks. The solid line refers to the MV efficient portfolios, the dashed line to MTE efficient portfolios. ${ }^{\star}$
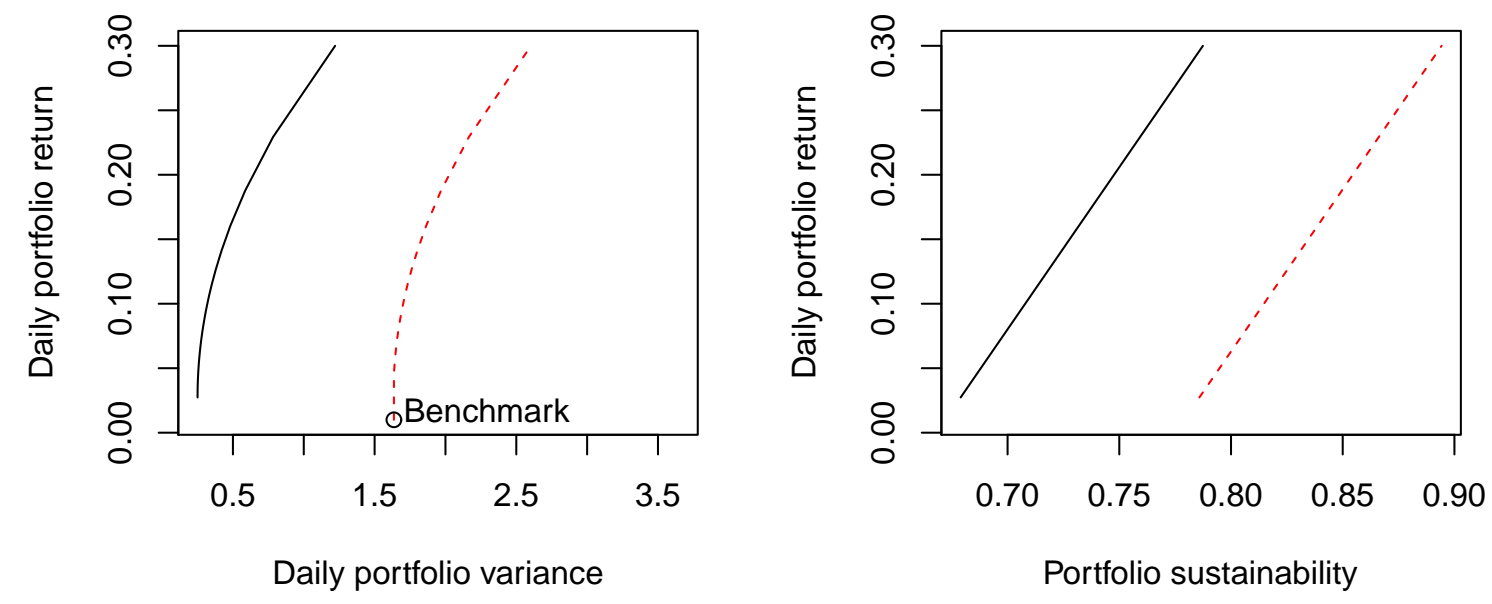

^ The daily portfolio return and variance are calibrated on the returns expressed in percentage points for the universe described in Section 3. 
An expression for the optimal weight vector for the MTE efficient investor in (2.2) is derived in the Appendix. With $b$ given in $(2.5)$ and $\gamma$ a $(N \times 1)$ vector containing the covariances between the benchmark return and the returns of the respective $N$ assets, we obtain

$$
\begin{gathered}
\omega_{\mathrm{MTE}}(\lambda)=a_{\mathrm{MTE}}+\frac{1}{\lambda} b \\
\text { with } a_{\mathrm{MTE}}=\Sigma^{-1}\left(\frac{\gamma\left(\iota^{\prime} \Sigma^{-1} \iota\right)-\iota\left(\iota^{\prime} \Sigma^{-1} \gamma\right)+\iota}{\iota^{\prime} \Sigma^{-1} \iota}\right) .
\end{gathered}
$$

Similar to the weights of an MV efficient portfolio in (2.4), the weight vector of the MTE efficient portfolio is given by the sum of two weight vectors $a_{\mathrm{MTE}}$ and $b$, weighted by one and $1 / \lambda$ respectively. The weight vector $a_{\mathrm{MTE}}$ corresponds to the minimum tracking error portfolio and the sum of the weights is one. The minimum tracking error portfolio is preferred by investors with the highest possible risk aversion level $\lambda=+\infty$. Interestingly, the second weight vector $b$ corresponds to exactly the same zero investment portfolio as in the MV case. In this case, the weight vector $b$ can be interpreted as the weight deviations needed to obtain an excess return on top of the minimum tracking error portfolio. The higher the factor $1 / \lambda$ then, the more weight is put on the zero investment portfolio $b$ and the higher the portfolio return and tracking error variance becomes.

For $\lambda \geq 0$, the portfolio return $\mu_{\mathrm{MTE}}(\lambda)=\omega_{\mathrm{MTE}}^{\prime}(\lambda) \mu$ and portfolio variance $\sigma_{\mathrm{MTE}}^{2}(\lambda)=$ $\omega_{\text {MTE }}^{\prime}(\lambda) \sum \omega_{\text {MTE }}(\lambda)$ of the MTE efficient portfolios also give rise to a hyperbola in the portfolio mean versus portfolio variance plane. This hyperbola is referred to as the MTE efficient frontier. The dashed line in the left panel of Figure 1 plots the MTE efficient frontier for the US constituents of the MSCI World index for the sample period 2003-2010, having the S\&P100 as benchmark (see Section 3 for more details on the data). In case the benchmark is an index based on a subset of the total invest- 
ment universe, the MTE efficient frontier has the benchmark as one of its points with corresponding tracking error volatility equal to zero. In case the benchmark does not lie on the MV efficient frontier, the MTE efficient frontier does not share any points with the MV efficient frontier. Moreover, the horizontal distance between the MV and MTE efficient frontier is the same for every target return level (see Roll (1992)), as shown in Figure 1.

\subsection{Linear relationship between the portfolio return and sus- tainability of the efficient portfolio}

We now focus on the relation between the portfolio return and the portfolio sustainability of the above efficient portfolios. The sustainability of an MV efficient portfolio is denoted by $\phi_{\mathrm{MV}}(\lambda)$. For MTE portfolios, we use a similar notation, i.e. $\phi_{\mathrm{MTE}}(\lambda)$. Note that

$$
\begin{aligned}
& \mu_{\mathrm{MV}}(\lambda)=\omega_{\mathrm{MV}}^{\prime}(\lambda) \mu=a_{\mathrm{MV}}^{\prime} \mu+\frac{1}{\lambda} b^{\prime} \mu, \\
& \phi_{\mathrm{MV}}(\lambda)=\omega_{\mathrm{MV}}^{\prime}(\lambda) \phi=a_{\mathrm{MV}}^{\prime} \phi+\frac{1}{\lambda} b^{\prime} \phi,
\end{aligned}
$$

and similarly for the MTE portfolio. Since both the portfolio return $\mu_{\mathrm{MV}}(\lambda)$ and portfolio sustainability $\phi_{\mathrm{MV}}(\lambda)$ are a linear function of $\frac{1}{\lambda}$, it is immediate to find

$$
\mu_{\mathrm{MV}}(\lambda)=\alpha_{\mathrm{MV}}+\beta_{\mathrm{MV}} \phi_{\mathrm{MV}}(\lambda)
$$

with

$$
\begin{aligned}
\alpha_{\mathrm{MV}} & =a_{\mathrm{MV}}^{\prime} \mu-a_{\mathrm{MV}}^{\prime} \phi \frac{b^{\prime} \mu}{b^{\prime} \phi}, \\
\text { and } \beta_{\mathrm{MV}} & =\frac{b^{\prime} \mu}{b^{\prime} \phi} .
\end{aligned}
$$


Alike the MV efficient portfolios, both the portfolio return and portfolio sustainability of the efficient MTE portfolio are a linear function of $\frac{1}{\lambda}$, which allows to write

$$
\mu_{\mathrm{MTE}}(\lambda)=\alpha_{\mathrm{MTE}}+\beta_{\mathrm{MTE}} \phi_{\mathrm{MTE}}(\lambda)
$$

with

$$
\alpha_{\mathrm{MTE}}=a_{\mathrm{MTE}}^{\prime} \mu-a_{\mathrm{MTE}}^{\prime} \phi \frac{b^{\prime} \mu}{b^{\prime} \phi}
$$

and $\beta_{\mathrm{MTE}}=\beta_{\mathrm{MV}}$. The interpretation of the linear relations (2.8) and (2.11) is as follows: for MV and MTE efficient portfolios the return increases or decreases linearly as a function of the sustainability along the efficient frontier. Interestingly, the slope of this linear relation, $\beta$, is identical for MV and MTE efficient portfolios. The right panel of Figure 1 illustrates this finding. The solid line plots the return for efficient MV portfolios as a function of portfolio sustainability. The dashed line corresponds to MTE efficient portfolios and is thus parallel to the solid line. In what follows, we refer to an investor demanding a minimum level of portfolio sustainability $\phi_{0}$ as a SRI-constrained investor.

The slope of the linear relation between $\mu(\lambda)$ and $\phi(\lambda)$ can be negative or positive. In case $\beta<0$, the SRI-constrained investor might not be able to choose efficient portfolios above a certain portfolio return $\mu_{0}$. In case $\beta>0$, as illustrated in the right panel of Figure 1, the SRI-constrained investor might not be able to choose efficient portfolios below a certain portfolio return $\mu_{0}$. This stems from the fact that efficient portfolios with a higher target return have a lower portfolio sustainability for $\beta<0$ and efficient portfolios with a higher target return have a higher portfolio sustainability for $\beta>0$. Graphically, it means that in the traditional mean-variance plane, the lower-left (upper-right) part of the efficient frontier would not be accessible for a SRI-constrained investor for $\beta>0(\beta<0)$. 


\subsection{Sustainability constrained efficient portfolios}

We now focus on the optimization problem for SRI-constrained MV and MTE investors. The SRI-constrained MV investor solves the problem:

$$
\max _{\omega} \omega^{\prime} \mu-\frac{\lambda}{2} \omega^{\prime} \Sigma \omega
$$

subject to: $\omega^{\prime} \iota=1$ and $\omega^{\prime} \phi \geq \phi_{0}$.

Compared to (2.1), the SRI-constrained investor thus faces the additional constraint that the portfolio sustainability level should be above a certain threshold level $\phi_{0}$. In case the sustainability level of the unconstrained problem $\omega_{\mathrm{MV}}^{\prime}(\lambda) \phi$ is larger than $\phi_{0}$ for a given $\lambda$, the solution remains unaltered obviously. In contrast, in case the sustainability constraint is binding, i.e. $\omega_{\mathrm{MV}}^{\prime}(\lambda) \phi \leq \phi_{0}$, the solution to (2.13) is given by

$$
\begin{gathered}
\omega_{\mathrm{MV}}\left(\lambda, \phi_{0}\right)=a_{\mathrm{MV}}+\frac{b}{\lambda}+\frac{c}{\lambda} l_{2}, \\
\text { with } c=\Sigma^{-1} \iota\left(\frac{\iota^{\prime} \Sigma^{-1} \phi}{\iota^{\prime} \Sigma^{-1} \iota}\right)-\Sigma^{-1} \phi \text { and } l_{2}=\frac{\lambda\left(\phi_{0}-a_{\mathrm{MV}}^{\prime} \phi-\frac{b^{\prime} \phi}{\lambda}\right)}{c^{\prime} \phi} .
\end{gathered}
$$

The weight vector now contains an extra zero investment portfolio $c$, on top of the components $a_{\mathrm{Mv}}$ and $b$ which are the same as in the weight vector of the unconstrained problem in (2.4).

Similarly, the SRI-constrained MTE investor solves the problem:

$$
\max _{\tilde{\omega}} \tilde{\omega}^{\prime} \tilde{\mu}-\frac{\lambda}{2} \tilde{\omega}^{\prime} \tilde{\Sigma} \tilde{\omega}
$$

subject to: $\tilde{\omega}^{\prime} \iota=0, \tilde{\omega}^{\prime} j=-1$ and $\omega^{\prime} \phi \geq \phi_{0}$.

In case the sustainability level $\omega_{\mathrm{MTE}}^{\prime}(\lambda) \phi$ is larger than $\phi_{0}$ for a given $\lambda$, the solution re- 
mains unaltered obviously. In contrast, in case the sustainability constraint is binding, i.e. $\omega_{\mathrm{MTE}}^{\prime}(\lambda) \phi \leq \phi_{0}$, the solution to $(2.16)$ is given by

$$
\begin{gathered}
\omega_{\mathrm{MTE}}\left(\lambda, \phi_{0}\right)=a_{\mathrm{MTE}}+\frac{b}{\lambda}+\frac{c}{\lambda} l_{2}, \\
\text { with } c=\Sigma^{-1} \iota\left(\frac{\iota^{\prime} \Sigma^{-1} \phi}{\iota^{\prime} \Sigma^{-1} \iota}\right)-\Sigma^{-1} \phi \text { and } l_{2}=\frac{\lambda\left(\phi_{0}-a_{\mathrm{MTE}}^{\prime} \phi-\frac{b^{\prime} \phi}{\lambda}\right)}{c^{\prime} \phi} .
\end{gathered}
$$

Only the vector $a$ is different in the solution of the constrained MTE problem in (2.17) compared to the solution of the constrained MV (2.14).

As first noted in Drut (2010), the extent to which the solution of the constrained and unconstrained problem differ, depends on the risk aversion level $\lambda$. Given the similarity between MV and MTE efficient portfolios, the discussion below focusses solely on the latter. Denote by $\lambda_{0, \mathrm{MTE}}$, the risk aversion level for which $\omega_{\mathrm{MTE}}^{\prime}\left(\lambda_{0, \mathrm{MTE}}\right) \phi=\phi_{0}$. The effect of a sustainability constraint on the efficient MV and MTE frontier can be subdivided in four cases:

(i) In case $\beta>0$, i.e. the sustainability increases with the target return, and $a_{\mathrm{MTE}}^{\prime} \phi \geq$ $\phi_{0}$, i.e. the sustainability level of the minimum tracking error portfolio is above the threshold, the unconstrained and constrained MTE efficient frontier coincide. This is shown in the upper left panel of Figure 2.

(ii) The constrained efficient frontier does not share any points with the unconstrained efficient frontier, which is illustrated in the upper right panel of Figure 2. The solid line refers to the unconstrained efficient frontier and the dotted line to the constrained efficient frontier. The constrained frontier lies below the unconstrained frontier, meaning SRI-constrained investors face a higher portfolio variance for a given target return (or a lower portfolio return for a given portfolio variance) due to the restriction on portfolio sustainability. This situation emerges when $\beta<0$ 
and $a_{\mathrm{MTE}}^{\prime} \phi<\phi_{0}$ for the MTE efficient frontier. This means that the sustainability decreases with the target return along the unconstrained efficient frontier and that the sustainability of the minimum tracking error portfolio is already below the threshold.

(iii) The left part of the unconstrained and constrained efficient frontier coincide, while the right part is distinct. This case is illustrated in the lower left panel of Figure 2. This situation emerges for the MTE efficient frontier when $\beta<0$ and $a_{\mathrm{MTE}}^{\prime} \phi>\phi_{0}$. In other words, the sustainability decreases with the portfolio return but the sustainability of the minimum tracking error portfolio is above the threshold level. The solution to both the unconstrained and constrained MTE optimization problem is given by (2.6) for $\lambda \geq \lambda_{0, \mathrm{MTE}}$. For smaller values of $\lambda$, the sustainability constraint becomes binding and the solution to the constrained MTE problem is given by (2.17).

(iv) Finally, the right part of the unconstrained and constrained efficient frontier coincide, while the left part is distinct, as can be seen on the lower right panel in Figure 2. This situation emerges for the MTE efficient frontier when $\beta>0$ and $a_{\mathrm{MTE}}^{\prime} \phi<\phi_{0}$. In other words, the sustainability increases with the portfolio return and the sustainability of the minimum tracking error portfolio is below the threshold level. The unconstrained and constrained MTE efficient frontier coincide for $\lambda \leq \lambda_{0, \mathrm{MTE}}$, while for larger values of $\lambda$, the solution for the MTE constrained problem is given by $(2.17)$.

\subsection{The performance loss due to sustainability constraint}

In some cases, the sustainability constraint leads to a shift in the efficient frontier. Using the explicit expressions for the portfolio weights of MV and MTE efficient portfolios 
Figure 2: Impact of a sustainability constraint on the efficient frontier in four scenarios. ${ }^{\star}$
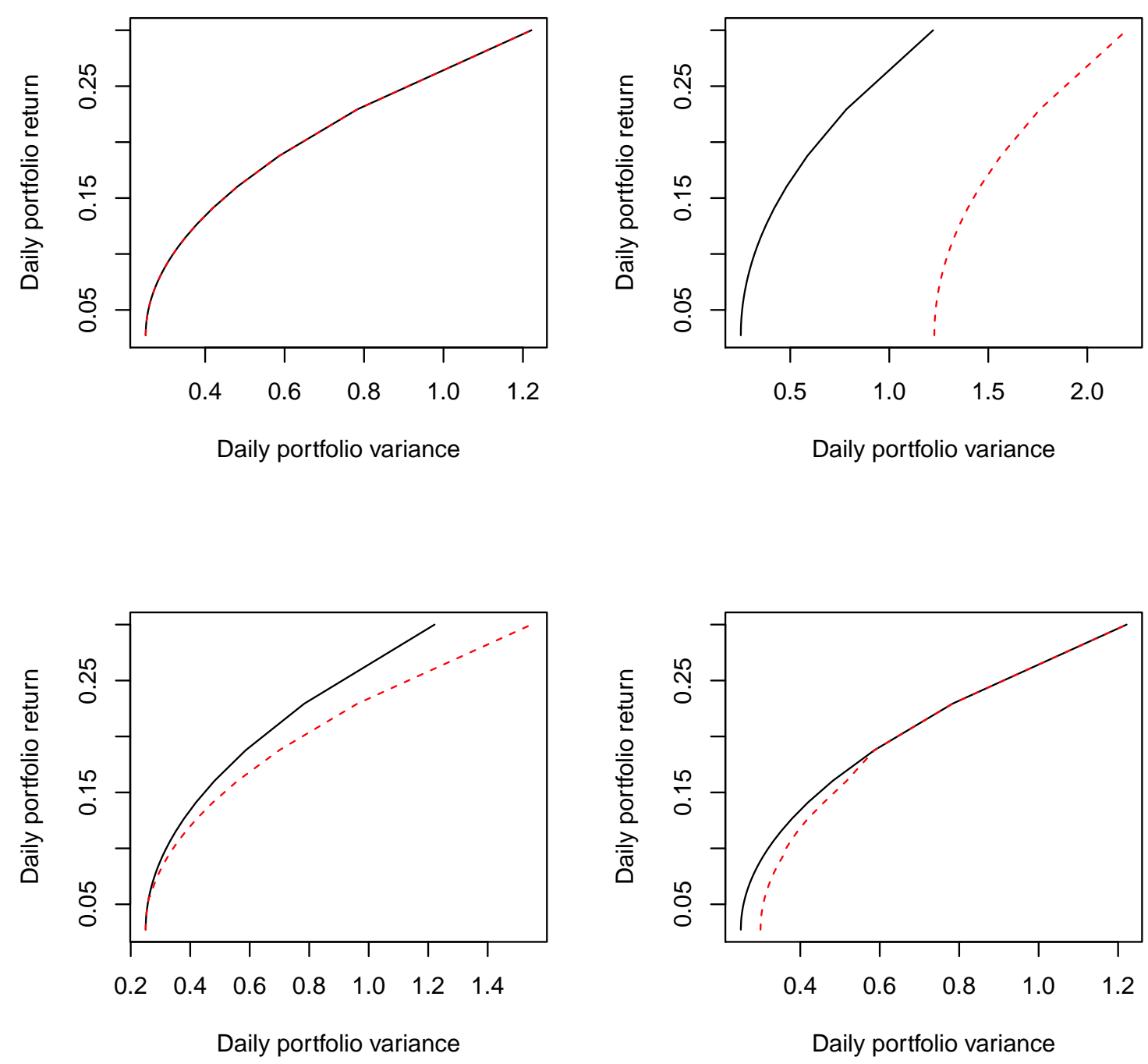

$\star$ Every panel plots the unconstrained efficient frontier (solid line) and the constrained efficient frontier (dotted). Mean and variances are reported for returns expressed in percentage points. 
of Subsection 2.3, we want to estimate (i) the increase in portfolio variance for a given target return, and (ii) the decrease in portfolio return for a given portfolio variance. Visually, in the mean-variance plane, (i) is the horizontal difference between the constrained and the unconstrained efficient frontier, whereas (ii) is the vertical difference between the constrained and unconstrained efficient frontier. We do this also for the mean-tracking error efficient investor.

The increase in portfolio variance or tracking error variance for a given target return. Denote by $\lambda_{\mu_{c}}$ the risk aversion parameter for which the portfolio return of the unconstrained MV efficient portfolio is $\mu_{c}$, i.e. $\mu_{c}=\omega_{\mathrm{MV}}^{\prime}\left(\lambda_{\mu_{c}}\right) \mu$. Define similarly $\lambda_{\mu_{c}}^{+}$as the risk aversion parameter for which the portfolio return of the portfolio constrained to have sustainability level $\phi_{0}$ is $\mu_{c}$, i.e. $\mu_{c}=\omega_{\mathrm{MV}}^{\prime}\left(\lambda_{\mu_{c}}^{+}, \phi_{0}\right) \mu$. The increase in portfolio variance a SRI-constrained MV investor faces compared to an unconstrained MV investor, can then be expressed as

$$
\Delta \sigma_{\mathrm{MV}}^{2}\left(\mu_{c}, \phi_{0}\right)=\left(\omega_{\mathrm{MV}}\left(\lambda_{\mu_{c}}^{+}, \phi_{0}\right)-\omega_{\mathrm{MV}}\left(\lambda_{\mu_{c}}\right)\right)^{\prime} \Sigma\left(\omega_{\mathrm{MV}}\left(\lambda_{\mu_{c}}^{+}, \phi_{0}\right)-\omega_{\mathrm{MV}}\left(\lambda_{\mu_{c}}\right)\right) .
$$

In other words, $\Delta \sigma_{\mathrm{MV}}^{2}\left(\mu_{c}, \phi_{0}\right)$ is the effect a sustainability restriction has on the MV efficient portfolio in terms of increased portfolio risk, for a given target return $\mu_{c}$ and sustainability threshold $\phi_{0}$.

Similarly, the increase in portfolio tracking error a SRI-constrained MTE efficient investor faces compared to an unconstrained MTE investor with the same target return $\mu_{c 2}$ is given by:

$$
\Delta \tilde{\sigma}_{\mathrm{MTE}}^{2}\left(\mu_{c 2}, \phi_{0}\right)=\left(\tilde{\omega}_{\mathrm{MTE}}\left(\lambda_{\mu_{c 2}}^{+}, \phi_{0}\right)-\tilde{\omega}_{\mathrm{MTE}}\left(\lambda_{\mu_{c 2}}\right)\right)^{\prime} \tilde{\Sigma}\left(\tilde{\omega}_{\mathrm{MTE}}\left(\lambda_{\mu_{c 2}}^{+}, \phi_{0}\right)-\tilde{\omega}_{\mathrm{MTE}}\left(\lambda_{\mu_{c 2}}\right)\right)
$$

where $\lambda_{\mu_{c 2}}$ the risk aversion parameter for which the portfolio return of the unconstrained MTE efficient portfolio is $\mu_{c 2}$ and $\lambda_{\mu_{c 2}}^{+}$is the risk aversion parameter for which 
the portfolio return of the portfolio constrained to have sustainability level $\phi_{0}$ is $\mu_{c 2}$.

The loss in portfolio return for a given variance or tracking error variance target. We now focus on the loss in portfolio return a SRI-constrained MV investor faces in comparison to a MV investor that doesn't face the sustainability restriction, keeping the portfolio variance fixed. Denote by $\lambda_{\sigma_{c}^{2}}$ the risk aversion parameter for which the portfolio variance of the unconstrained MV efficient portfolio is $\sigma_{c}^{2}$, i.e. $\sigma_{c}^{2}=$ $\omega_{\mathrm{MV}}^{\prime}\left(\lambda_{\sigma_{c}^{2}}\right) \sum \omega_{\mathrm{MV}}\left(\lambda_{\sigma_{c}^{2}}\right)$. Similarly, denote by $\lambda_{\sigma_{c}^{2}}^{+}$the risk aversion parameter for which the portfolio variance of the portfolio constrained to have sustainability level $\phi_{0}$ is $\sigma_{c}^{2}$, i.e. $\sigma_{c}^{2}=\omega_{\mathrm{MV}}^{\prime}\left(\lambda_{\sigma_{c}^{2}}^{+}, \phi_{0}\right) \sum \omega_{\mathrm{MV}}\left(\lambda_{\sigma_{c}^{2}}^{+}, \phi_{0}\right)$. In case the sustainability constraint is binding, the loss in portfolio return for a sustainability threshold $\phi_{0}$ and target variance $\sigma_{c}^{2}$ is then given by:

$$
\Delta \mu_{\mathrm{MV}}\left(\sigma_{c}^{2}, \phi_{0}\right)=\left(\omega_{\mathrm{MV}}\left(\lambda_{\sigma_{c}^{2}}^{+}, \phi_{0}\right)-\omega_{\mathrm{MV}}\left(\lambda_{\sigma_{c}^{2}}\right)\right)^{\prime} \mu .
$$

In other words, $\Delta \mu_{\mathrm{MV}}\left(\sigma_{c}^{2}, \phi_{0}\right)$ is the effect a sustainability restriction has on the MV efficient portfolio in terms of foregone portfolio return, for a given target variance level $\sigma_{c}^{2}$ and sustainability threshold $\phi_{0}$.

The loss in portfolio return a SRI-constrained MTE investor faces in comparison to a MTE investor that doesn't face the sustainability restriction, keeping the portfolio tracking error variance fixed at $\tilde{\sigma}_{c}^{2}$ is given by:

$$
\Delta \mu_{\mathrm{MTE}}\left(\tilde{\sigma}_{c}^{2}, \phi_{0}\right)=\left(\omega_{\mathrm{MTE}}\left(\lambda_{\tilde{\sigma}_{c}^{2}}^{+}, \phi_{0}\right)-\omega_{\mathrm{MTE}}\left(\lambda_{\tilde{\sigma}_{c}^{2}}\right)\right)^{\prime} \mu,
$$

where $\lambda_{\tilde{\sigma}_{c}^{2}}$ the risk aversion parameter for which the portfolio tracking error variance of the unconstrained MTE efficient portfolio is $\tilde{\sigma}_{c}^{2}$, i.e. $\tilde{\sigma}_{c}^{2}=\tilde{\omega}_{\mathrm{MTE}}^{\prime}\left(\lambda_{\tilde{\sigma}_{c}^{2}}\right) \tilde{\Sigma} \tilde{\omega}_{\mathrm{MTE}}\left(\lambda_{\tilde{\sigma}_{c}^{2}}\right)$. Similarly, $\lambda_{\tilde{\sigma}_{c}^{2}}^{+}$is the risk aversion parameter for which the portfolio tracking error variance of the constrained portfolio is $\tilde{\sigma}_{c}^{2}$, i.e. $\tilde{\sigma}_{c}^{2}=\tilde{\omega}_{\mathrm{MTE}}^{\prime}\left(\lambda_{\tilde{\sigma}_{c}^{2}}^{+}, \phi_{0}\right) \tilde{\Sigma} \tilde{\omega}_{\mathrm{MTE}}\left(\lambda_{\tilde{\sigma}_{c}^{2}}^{+}, \phi_{0}\right)$. 


\section{Data}

The investment universe consists of the US constituents of the MSCI World index. The size of the universe varies between $N=470$ stocks in 2003 up to a maximum of $N=638$ stocks in 2010.

Daily returns are computed as $r_{t}=100\left(P_{t}-P_{t-1}\right) / P_{t-1}$, with $P_{t}$ the total return index of the stock on day $t$ (retrieved from Datastream). The sample period ranges from January 2003 up to December 2010. The S\&P 100 index is used as the benchmark in the tracking error optimized portfolios.

Table 1 reports the descriptive statistics for the assets in the considered investment universe per time frame and industry, and compares them with the returns on the S\&P 100 index. We see that the average return across stocks is in line with the average daily return of the index. The impact of the financial crisis in 2008 explains the negative average return for that year and the negative return of the banking sector over the whole period. The cross-sectional minimum and maximum statistics illustrate the variation in average return and variance across stocks.

A critical input in characterizing the sustainability of MV and MTE efficient portfolios is of course the methodology used to calculate each stock's sustainability. A popular approach in academic research is to use the database of KLD Research \& Analytics, Inc. An aggregate sustainability score per asset is then created by simply taking the average over all available ratings on various sustainability aspects per asset (see e.g. Herzel et al. (2011), Galema et al. (2008) and Statman and Gkushkov (2009)).

In practice, funds often construct their sustainability score based on questionnaire data bought from one or more external parties. Then, a committee of sustainability experts determines a procedure to obtain a total sustainability score based on the specific aspects related to sustainability. The aggregation of the raw sustainability data to a single score per asset is thus in both cases done in a relatively arbitrary way. 
Table 1: Descriptive statistics of the returns of the assets in the universe. ${ }^{\star}$

\begin{tabular}{lccccccccc}
\hline & Number of assets & \multicolumn{3}{c}{ Mean of daily returns } & \multicolumn{2}{c}{ Variance of daily returns } & \multicolumn{2}{c}{ S\&P 100 } \\
\hline Full dataset & & Min & Average & Max & Min & Average & Max & Mean & Variance \\
2003 & 330 & -0.30 & 0.02 & 0.18 & 1.18 & 5.69 & 61.25 & 0.01 & 1.70 \\
2004 & 334 & -0.12 & 0.11 & 0.49 & 0.69 & 3.81 & 23.91 & 0.07 & 1.18 \\
2005 & 459 & -0.21 & 0.05 & 0.42 & 0.52 & 2.59 & 16.01 & 0.02 & 0.49 \\
2006 & 492 & -0.25 & 0.03 & 0.37 & 0.54 & 2.39 & 15.68 & 0.00 & 0.38 \\
2007 & 539 & -0.29 & 0.04 & 0.35 & 0.40 & 2.57 & 15.39 & 0.06 & 0.36 \\
2008 & 577 & -0.52 & -0.00 & 0.84 & 0.50 & 3.39 & 22.89 & 0.01 & 0.99 \\
2009 & 632 & -2.06 & -0.24 & 0.18 & 2.75 & 20.15 & 326.74 & -0.18 & 6.38 \\
2010 & 638 & -2.04 & 0.11 & 0.62 & 0.45 & 14.56 & 448.18 & 0.07 & 2.59 \\
IT Hardware & 638 & -0.77 & 0.06 & 0.43 & 0.57 & 5.05 & 382.29 & 0.04 & 1.18 \\
Banks & 31 & -0.03 & 0.03 & 0.18 & 3.84 & 6.58 & 13.13 & & \\
General Retailers & 19 & -0.07 & -0.02 & 0.03 & 5.60 & 10.81 & 19.37 & & \\
Health & 23 & -0.05 & 0.03 & 0.11 & 1.68 & 5.63 & 16.73 & & \\
Support Services & 17 & -0.05 & 0.02 & 0.05 & 1.78 & 3.54 & 5.89 & & \\
\hline
\end{tabular}

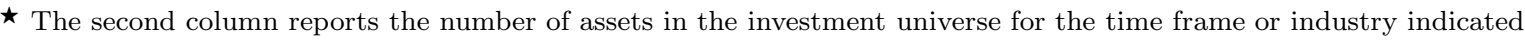
the first column. The following six columns report the cross-sectional minimum, mean and maximum statistics for the time series mean and variance of the daily return (in percentage points). The last two columns are the time series mean and variance of the daily return (in percentage points) of the $\mathrm{S} \& \mathrm{P} 100$ index.

In contrast, a data-driven procedure was proposed in Hosseini and Kaneko (2011). They use principal component analysis (PCA) to construct one sustainability rating per country based on many underlying sustainability indicators, obtained via external parties. This approach has the disadvantage of giving possibly negative weights to sustainability indicators. We adapt this method such that it does not have this shortcoming.

Our questionnaire database is from Eiris, an independent not-for-profit organization that rates companies on environmental, social, governance and other ethical aspects. The Eiris database contains the answers to more than 400 questions related to sustainability. The database is updated on a yearly basis and the sample period runs from 2003 up to 2010. As a first step, we only retain questions for which we have a record for at least $75 \%$ of the companies. Of the remaining 25 questions, we remove one question since it contains the same answer for all companies. The $Q=24$ retained questions are listed in the third column of Table 2. Except for the first two questions, 
Table 2: Selected Eiris questions and their average weight for the construction of the total sustainability score. ${ }^{\star}$

\begin{tabular}{llc}
\hline & Question & Weight \\
\hline 1 & In how many countries listed in EIRIS Category B does the company have operations? & 0.000 \\
2 & In how many countries listed in EIRIS Category A does the company have operations? & 0.000 \\
3 & Does the company have policies and procedures on bribery and corruption? & 0.043 \\
4 & What level of engagement with stakeholders is disclosed by the company? & 0.061 \\
5 & Does the company have policies on maintaining good relations with customers and/or suppliers? & 0.040 \\
6 & How clear is the evidence of systems to maintain good relations with customers and/or suppliers? & 0.051 \\
7 & How good is the company's policy on equal opportunity and diversity issues? & 0.058 \\
8 & How clear is the evidence of systems and practices to support equal opportunities and diversity? & 0.067 \\
9 & How clear is the evidence of health \& safety systems? & 0.065 \\
10 & How clear is the evidence of systems to manage employee relations? & 0.026 \\
11 & How clear is the evidence of systems to support employee training and development? & 0.051 \\
12 & How clear is the evidence of systems and practices to advance job creation and security? & 0.025 \\
13 & How clear is the company's commitment to community or charitable work? & 0.070 \\
14 & How good are the company's policies towards its stakeholders overall? & 0.075 \\
15 & How good are the company's management systems for stakeholders overall? & 0.082 \\
16 & How many stakeholder issues have been allocated to board members? & 0.057 \\
17 & How good is the company's quantitative reporting on stakeholder relationships? & 0.074 \\
18 & How many of the company's directors are women? & 0.030 \\
19 & How many of the core elements of corporate governance does the company have? & 0.000 \\
20 & How good is the company's code of ethics? & 0.048 \\
21 & How good is company's system for implementing its code of ethics? & 0.053 \\
22 & Does the company separate the roles of chairman and chief executive? & 0.001 \\
23 & Are more than 33\% of the company board independent non-executives? & 0.015 \\
24 & Does the company have an audit committee with a majority of independent directors? & 0.008 \\
\hline
\end{tabular}

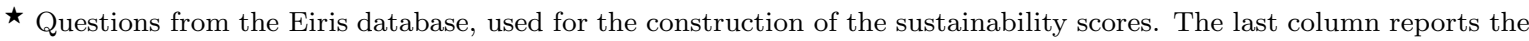
average weight these questions have in the construction of the sustainability score over the years 2003 until 2010.

the answers in the Eiris database are categorical variables. We first transform these into numerical values in the following way: "1" indicates the lowest sustainability level, "2" a higher sustainability level, and so on. We then use PCA to reduce the dimension of the retained Eiris dataset (see Table 2) to one sustainability rating per asset, while preserving as much as possible of the variation in the data set. However, since higher values of the answers indicate a higher level of sustainability, we require the loadings of the principal component to be non-negative. This ensures that the principal component represents a global measure of the total sustainability of that asset. Furthermore, we make sure the loadings sum to one such that the loadings can be interpreted as a percentage of the individual variables contribution to the total score. 
More formally, let $X_{t}$ be the $Q \times N_{t}$ matrix with standardized input data for year $t$, with $Q$ the number of retained questions and $N_{t}$ the number of assets in year $t$. We determine the $Q \times 1$ vector of weights $v_{t}^{*}$, such that

$$
v_{t}^{*}=\operatorname{argmax}_{v} \operatorname{Var}\left(v^{\prime} X_{t}\right), \text { with } v \geq 0 \text { and } v^{\prime} \iota=1,
$$

with $\iota$ the $Q \times 1$ vector of ones. The vector with sustainability scores for all assets in year $t$ is then given by $\phi_{t}=X_{t}^{\prime} v_{t}^{*}$. In other words, the total sustainability scores are a weighted average of the scores on various sustainability aspects, such that the variance of the total sustainability scores is maximal. We apply this procedure on the Eiris dataset and report in the second column of Table 2 the weight $v_{t}^{*}$ averaged over $t=2003, \ldots, 2010$. We drop the subscript $t$ in what follows, for the ease of notation.

The vector of the sustainability scores of the $N$ assets is denoted by $\phi=\left(\phi_{1}, \ldots, \phi_{N}\right)^{\prime}$, with $\phi_{i}$ the sustainability score of the $i$-th asset. As a final step, we map the values of $\phi$ to the interval $[0,1]$, to make the interpretation of $\phi$ more intuitive. We replace each value of $\phi_{i}$, for $i=1, \ldots, N$, by the corresponding quantile. For example, if an asset has a sustainability score of 0.3 , then $30 \%$ of the assets in the investment universe are less sustainable and $70 \%$ is of the assets are more sustainable.

In Figure 3 the self constructed questionaire-based sustainability scores are compared to the ones of two external parties. The first external party is $\mathrm{KBC}$, a Belgian bank, that also constructs sustainability scores based on the information in the Eiris database. In contrast to our methodology, KBC assigns a sustainability score to each asset based on an analysis by a committee of sustainability experts. The second external party, Vigeo, an organization owned by financial institutions, trade unions and corporates, collects its own sustainability data, different from the Eiris ones. On top of that, Vigeo calculates a single sustainability score per asset, based on their own 
Figure 3: Pairwise scatterplots of the sustainability scores provided by KBC, Vigeo and the self-constructed scores based on the Eiris dataset.
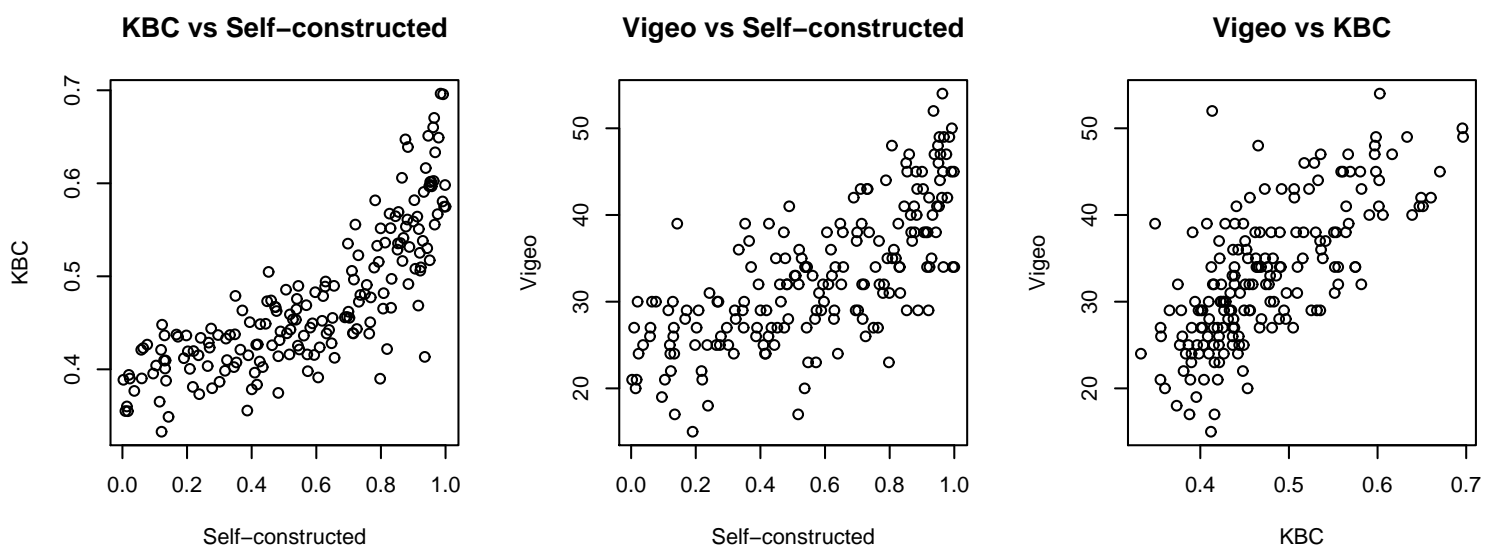

procedure designed by sustainability experts.

For the year 2010, we select the subset of 187 US companies for which we have sustainability scores from all three parties. Figure 3 plots a pair-by-pair comparison of the 3 different sustainability scores. We observe a clear linear relation between each pair sustainability scores, indicating they are measuring the same underlying construct, although there is substantial noise. The Pearson correlations between the pairs (Self-constructed,KBC), (Self constructed,Vigeo) and (KBC,Vigeo) are respectively: $80 \%, 72 \%$ and $71 \%$. The corresponding Spearman correlations are $84 \%, 73 \%$ and $71 \%$ respectively. The fact that the Spearman correlation is larger than the Pearson correlation for the Self-constructed scores versus the KBC scores, indicates that the relation between the Self-constructed sustainability scores and the KBC sustainability scores is to a greater extent monotone than linear, which is confirmed by a visual inspection of the left panel of Figure 3. We conclude that the scores of different parties have a large degree of similarity. Since we do not want our results to be influenced by subjective choices of external providers of sustainability scores, we proceed with the self-constructed scores based on our data-driven methodology. 


\section{Estimation procedure}

The expected mean return vector $\mu$ is estimated by the sample mean. In our application, the number of assets $(N)$ is large compared to the number of time series observations $(T)$. We therefore use the Ledoit and Wolf (2003, 2004b) procedure of data-driven shrinking the sample covariance matrix to the equicorrelation matrix. This procedure is summarized in Appendix. By shrinking the sample covariance matrix towards the more structured estimator, the estimation error in the optimization is substantially reduced. Furthermore, it has the advantage that the covariance estimate is still invertible in case $N>T$. The inverse of the covariance matrix is important, since it is needed for e.g. the calculation of the portfolio weights in (2.4) and (2.6).

The $\alpha_{\mathrm{MV}}, \alpha_{\mathrm{MTE}}$ and $\beta$ in (2.9), (2.12) and (2.10) are estimated by replacing $\Sigma$ and $\mu$ by their respective estimates $\hat{\mu}$ and $\hat{\Sigma}$. We use the block bootstrapping methodology for the construction of the confidence regions around the estimated parameters of interest. The block bootstrap method takes into account the dynamic dependence in the returns, and in particular the conditional heteroscedasticity. An advantage of the block bootstrap method is that we don't have to assume a particular model for the volatility (Ruiz and Pascual, 2002). In particular, we apply the stationary bootstrap of Politis and Romano (1994), who suggest to resample blocks of data with random lengths, where the length of each block has a geometric distribution. We calibrate the

method such that the expected block length is 20, i.e. approximately one month of return data. We resample blocks of return vectors in order to keep the correlation between the different assets and create $B=1000$ bootstrap samples to construct the bootstrapped confidence intervals. The bounds of the pointwise confidence intervals are constructed using the bias-corrected accelerated percentile method. We explain this procedure briefly in Appendix and refer to DiCiccio and Romano (1988) for more details. 
The estimation and bootstrap methods are also useful for testing whether the efficient portfolio with target return $\mu_{c}$ has a sustainability level that is above the required threshold value $\phi_{0}$. Using the linear relation between the portfolio sustainability level $\phi_{\mathrm{MV}}(\lambda)$ and the portfolio return $\mu_{\mathrm{MV}}(\lambda)$ in $(2.8)$, the sustainability of a portfolio with target return $\mu_{c}$ can be written as $\phi_{\mathrm{MV}}=\left(\mu_{c}-\alpha_{\mathrm{MV}}\right) / \beta$. The corresponding one-sided hypothesis test is thus

$$
\mathrm{H}_{0}:\left(\mu_{c}-\alpha_{\mathrm{MV}}\right) / \beta \geq \phi_{0} \text { versus } \mathrm{H}_{a}:\left(\mu_{c}-\alpha_{\mathrm{MV}}\right) / \beta<\phi_{0} .
$$

Similarly, for the MTE efficient portfolio, we want to test

$$
\mathrm{H}_{0}:\left(\mu_{c}-\alpha_{\mathrm{MTE}}\right) / \beta \geq \phi_{0} \text { versus } \mathrm{H}_{a}:\left(\mu_{c}-\alpha_{\mathrm{MTE}}\right) / \beta<\phi_{0} .
$$

The corresponding test statistics are obtained by replacing the unknown quantities $\alpha_{\mathrm{MV}}$ and $\beta$ with their estimates. The test statistic for the test in (4.1) is thus given by $\hat{\phi}_{\mathrm{MV}_{c}}=\left(\mu_{c}-\hat{\alpha}_{\mathrm{MV}_{c}}\right) / \hat{\beta}$, and for the test in (4.2) the test statistic is given by $\hat{\phi}_{\mathrm{MTE}_{c}}=$ $\left(\mu_{c}-\hat{\alpha}_{\mathrm{MTE}_{c}}\right) / \hat{\beta}$.

Intuitively, the tests in (4.1) and (4.2) check whether an investor with a given target return $\mu_{c}$ and sustainability threshold $\phi_{0}$, has access to the mean-variance or meantracking error efficient portfolio, respectively. If the null hypothesis is rejected, the sustainability of the efficient portfolio is significantly below the level of sustainability required by the investor. In those cases the investor will pay a price for his sustainability constraint in terms of a higher risk and/or lower return. 


\section{$5 \quad$ Results}

In this section, we investigate empirically the interrelation between portfolio sustainability and portfolio performance, based on the financial return data and the sustainability scores discussed in Section $3 .{ }^{1}$ As a first step, we focus on the unconstrained MV and MTE efficient portfolios. We estimate the parameters $\alpha_{\mathrm{MV}}, \alpha_{\mathrm{MTE}}$ and $\beta$ and provide confidence regions around the reported results. We also apply the tests in (4.1) and (4.2) to evaluate whether the portfolio sustainability for the efficient MV and MTE portfolios is above a certain threshold. As a second step, we examine the cost a SRI-constrained investor faces in terms of foregone portfolio return and increased portfolio risk, in case a restriction on portfolio sustainability is enforced and binding. We report results for the total sample period, per year and per industry respectively. As discussed in Section 3, the entire sample period for assets in our investment universe runs from 2003-2010 for which the sample contains 2088 daily returns. Recall from Section 3 that the estimates are based on the returns expressed in percentage points. The sustainability scores updated on a yearly basis.

Table 3 reports the estimates of $\alpha_{\mathrm{MV}}(2.9), \alpha_{\mathrm{MTE}}(2.12)$ and $\beta$ (2.10) in the first three columns respectively. The next 6 columns report the upper and lower bounds of the respective bootstrapped confidence intervals. The sample used for the estimation is indicated for each row at the left of the Table 3 .

We find in Table 3 that the sign of $\hat{\beta}$ depends both on the time period and the industry. Recall from (2.8) and (2.11), that $\beta$ refers to the slope of the linear relation between the portfolio return and portfolio sustainability. Thus, the interpretation of

\footnotetext{
${ }^{1}$ In unreported results we have evaluated the robustness of our empirical findings on two accounts. First, we investigated mean tracking error efficient portfolios having the S\&P500 instead of the S\&P100 as benchmark to track. Second, we considered the sustainability scores of two external parties - Vigeo and KBC - as input instead of the data-driven sustainability scores we constructed based on the Eiris database. Our main empirical findings are robust to the choice of the benchmark and the sustainability scores.
} 
Table 3: Point estimates and 95\% confidence bands for the parameters in the linear relation between portfolio return and portfolio sustainability. ${ }^{\star}$

\begin{tabular}{rrrrrrrrrr}
\hline & $\hat{\alpha}_{\mathrm{MV}}$ & $\hat{\alpha}_{\mathrm{MTE}}$ & $\hat{\beta}$ & \multicolumn{2}{c}{$\hat{\alpha}_{\mathrm{MV}}$} & \multicolumn{2}{c}{$\hat{\alpha}_{\mathrm{MTE}}$} & \multicolumn{3}{c}{$\begin{array}{l}\text { lower } \\
\text { upper }\end{array}$} \\
\hline full dataset & -1.68 & -1.95 & 2.51 & -34.10 & 13.53 & -32.80 & 22.16 & -22.23 & 41.43 \\
2003 & -0.58 & -0.68 & 1.07 & -630.89 & 17.47 & -684.14 & 18.52 & -28.63 & 1100.42 \\
2004 & 0.98 & 1.45 & -1.81 & -641.06 & 1.64 & -835.36 & 2.10 & -2.87 & 1201.09 \\
2005 & -5.39 & -7.19 & 9.80 & -213.38 & 27.21 & -390.48 & 17.81 & -29.55 & 445.81 \\
2006 & -0.97 & -1.37 & 2.06 & -3.38 & 8318.95 & -4.31 & 9756.33 & -14943.76 & 6.71 \\
2007 & 2.21 & 2.90 & -3.82 & -160.31 & 76.03 & -236.60 & 93.07 & -143.19 & 364.37 \\
2008 & 1.20 & 2.01 & -2.82 & -5136.23 & 1.59 & -6343.63 & 2.30 & -2.95 & 9533.94 \\
2009 & -23.68 & -34.90 & 47.56 & -1994.02 & -8.40 & -2183.93 & -12.41 & 17.17 & 3468.86 \\
2010 & 0.69 & 0.97 & -1.28 & -577.59 & 1.81 & -653.03 & 2.01 & -3.15 & 937.42 \\
Banks & -0.23 & -0.27 & 0.54 & -11.34 & 15.46 & -12.00 & 10.70 & -15.71 & 20.51 \\
IT Hardware & 1.15 & 1.23 & -1.55 & -1.97 & 358.23 & -1.81 & 341.77 & -448.29 & 2.39 \\
General Retailers & 0.15 & 0.15 & -0.23 & -23.43 & 6.99 & -21.57 & 6.62 & -11.35 & 38.93 \\
Health & -0.60 & -0.54 & 0.90 & -106.02 & 0.75 & -35.68 & 1.57 & -2.44 & 55.54 \\
Support Services & 0.16 & 0.13 & -0.21 & -633.70 & 0.50 & -525.59 & 0.41 & -0.68 & 875.24 \\
\hline
\end{tabular}

$\star$ The estimates of $\alpha_{\mathrm{MV}}, \alpha_{\mathrm{MTE}}$ and $\beta$, are based on the sample indicated in the left column, with returns expressed in percentage points. The pointwise bootstrapped confidence bounds are constructed using 1000 bootstrap samples with $\alpha=0.05$

e.g. $\hat{\beta}=2.51$ for the entire sample, is that an increase in portfolio sustainability with one percentage point, corresponds to an estimated increase in the daily portfolio return (in percentage points) with 0.0251 for MV and MTE efficient portfolios. However, for almost all time periods and for all industries, the $\hat{\beta}$ is not significantly different from zero. By consequence, it is unclear whether the sustainability increases or decreases with the expected return along the MV and MTE efficient frontier. The year 2009 is an exception, where we find that $\hat{\beta}=47.56$ in percentage points is significantly different from zero. The confidence regions around $\alpha_{\mathrm{MV}}$ and $\alpha_{\mathrm{MTE}}$ are often extremely wide and almost always contain zero. Only in 2009, the parameters $\hat{\alpha}_{\mathrm{MV}}$ and $\hat{\alpha}_{\mathrm{MTE}}$ are significantly different from zero.

Figure 4 plots the estimated portfolio sustainability $\left(\mu_{c}-\hat{\alpha}_{\mathrm{MV}}\right) / \hat{\beta}$ and $\left(\mu_{c}-\hat{\alpha}_{\mathrm{MTE}}\right) / \hat{\beta}$ and corresponding confidence interval, as a function of the target return level $\mu_{c}$. The more to the right of the plot, the higher the target return becomes which corresponds to a lower risk aversion level, evidently. Efficient MV and MTE portfolios with a higher 
Figure 4: Point estimates and 95\% confidence bands of the sustainability level of MV and MTE efficient portfolios, as a function of the target return level (in percentage points).

Efficient MV portfolios

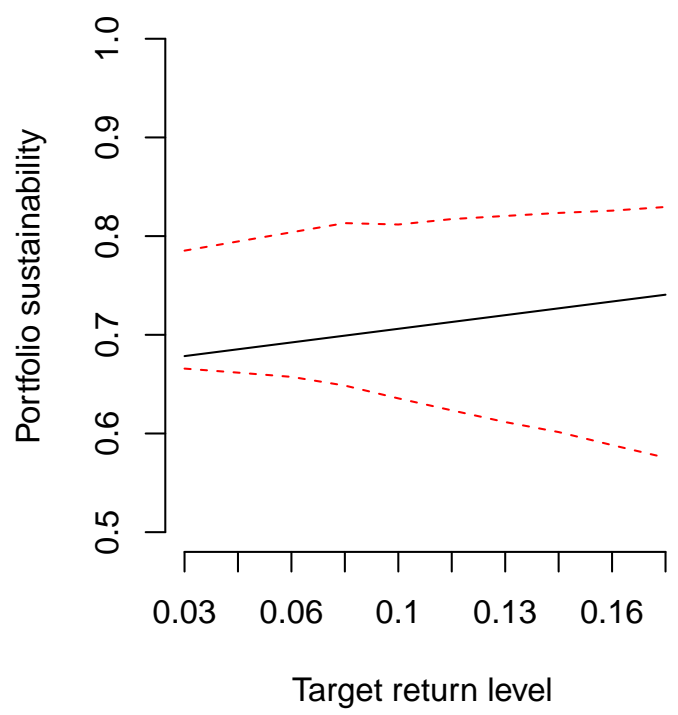

Efficient MTE portfolios

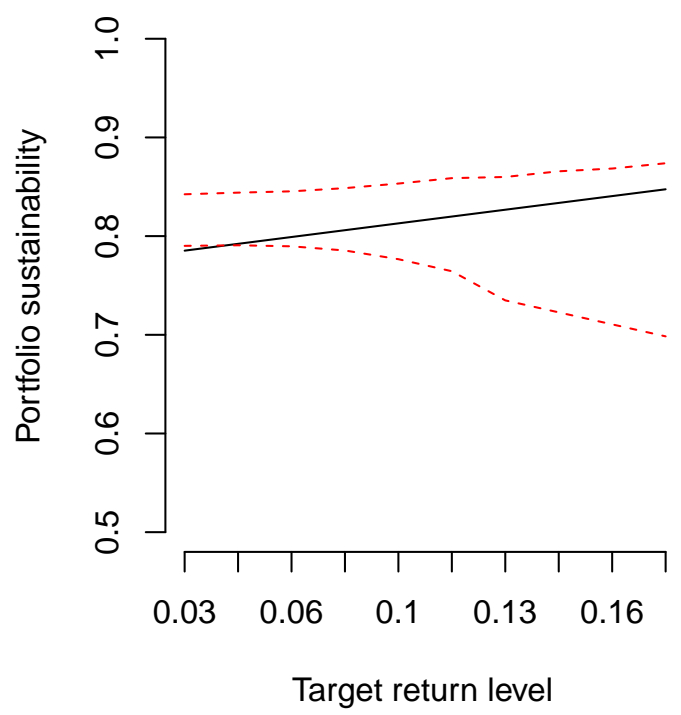

target return have a slightly higher portfolio sustainability, as illustrated on the plot. Note that for the equal target returns, the sustainability of the MTE efficient portfolios is somewhat higher than for MV efficient portfolios.

Most importantly, a visual inspection of the left and right panel in Figure 4 allows to evaluate the tests proposed in (4.1) and (4.2) respectively. We now focus on the right panel of Figure 4 to evaluate the test in (4.2). In case of $\phi_{0}=0.85$, the null hypothesis is rejected for daily target return levels up to around $0.11 \%$, since $\phi_{0}$ is above the highest confidence bound up to that point. For higher target return levels, $\phi_{0}$ is within the confidence region and the null hypothesis cannot be rejected. This implies that an SRI-constrained investor with sustainability threshold $\phi_{0}=0.85$, cannot invest in portfolios on the unconstrained MTE efficient frontier if his daily target return is below $0.11 \%$. 
Figure 5: Point estimates and 95\% confidence intervals for the change in variance and return due to imposing the minimum $90 \%$ sustainability target on the frontier of MV efficient portfolios. ${ }^{\star}$
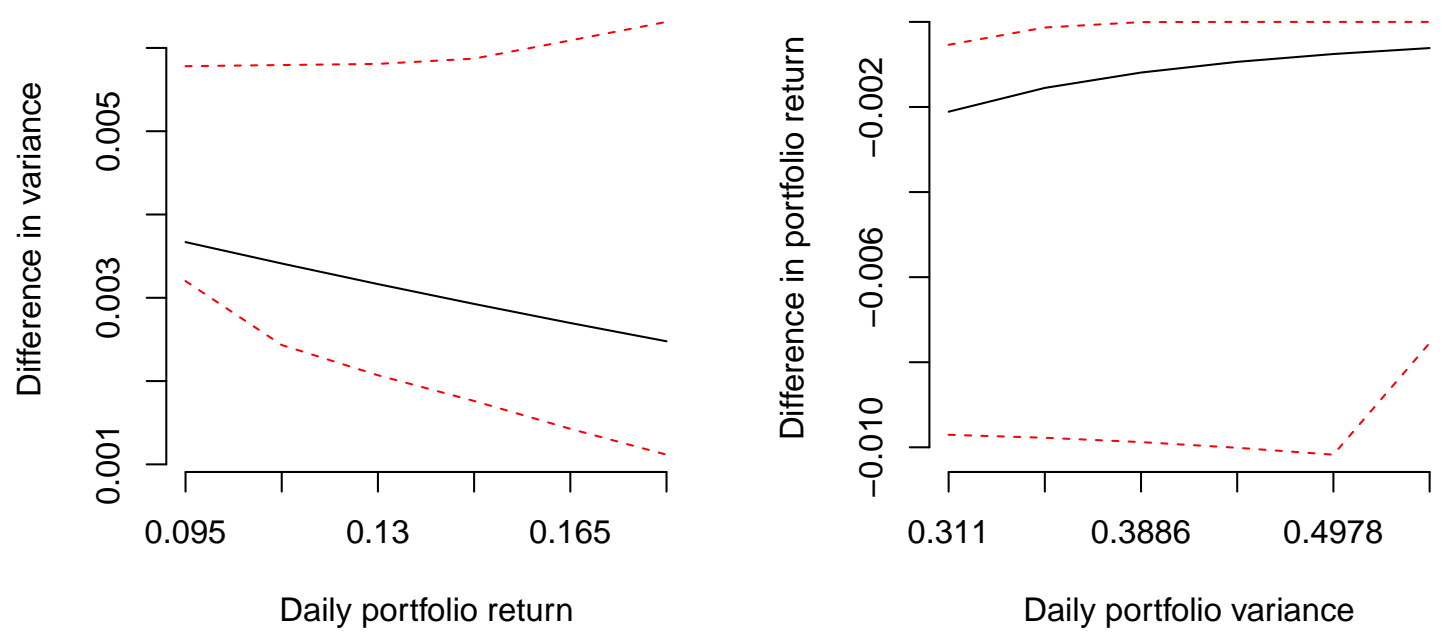

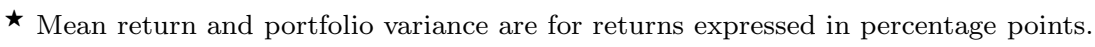

In Figure 5 we study the performance impact of imposing a sustainability target of $\phi_{0}=0.9$ on the MV efficient frontier, based on all stocks and the entire sample period. Since for this universe the sustainability increases with the target return along the unconstrained efficient frontier $(\hat{\beta}>0$ in Table 3$)$, we find that as expected, the performance impact is the biggest for the low return target (low variance) portfolio. Consider the unconstrained MV portfolio with mean return, variance and sustainability of $0.095,0.311$ and 0.71 , respectively. For this portfolio, the increase in variance due to the sustainability constraint is around 0.004 , which corresponds to a a relative increase in daily variance of about $2 \%$. The estimated difference in portfolio return is around -0.002. This corresponds to a relative decrease in portfolio return of more than $8 \%$ when moving from the unconstrained to the constrained efficient frontier. For higher target returns, the difference in return and variance becomes even smaller. 
Figure 6 plots the increase in variance and decrease in portfolio return due to the sustainability target of $\phi_{0}=0.9$ on the MTE efficient frontier. The unconstrained MTE portfolio with return target of 0.095 has a tracking error of 0.223 and sustainability level of 0.82 . The solid line in the left panel plots the estimated difference in tracking error volatility between the unconstrained and the constrained MTE efficient portfolios as a function of the target return $\mu_{c 2}$, i.e. $\Delta \hat{\tilde{\sigma}}_{\mathrm{MTE}}^{2}\left(\mu_{c 2}, \phi_{0}\right)$. We find that the difference in tracking error volatility is extremely small. The solid line in the right panel of Figure 6 plots the estimated difference in portfolio return between the unconstrained and the constrained MTE efficient portfolio as a function of its tracking error variance $\tilde{\sigma}_{c}^{2}$, i.e. $\Delta \hat{\mu}_{\mathrm{MTE}}\left(\tilde{\sigma}_{c}^{2}, \phi_{0}\right)$. We find that the impact of the sustainability constraint in terms of foregone portfolio return on MTE efficient portfolios is quite small, and not significantly different from zero for almost all values of the target tracking error.

In conclusion, it is interesting to note that for the considered sample and sample period a sustainability constraint of $\phi_{0}=0.9$ has a distinct impact on MV and MTE efficient portfolios. For MV efficient portfolios investors can face a substantial decrease in portfolio return. In contrast, the sustainability constraint barely has an effect on the MTE efficient frontier.

\section{Conclusion}

This research characterizes the sustainability of the mean-variance (MV) and meantracking error (MTE) efficient portfolios and studies the impact on the portfolio performance of constraints on the portfolio sustainability. We showed that for MV and MTE efficient portfolios a linear relation exists between portfolio sustainability and portfolio return. If the slope of this relation is negative, it implies that a higher target portfolio return will correspond to a lower portfolio sustainability. Therefore, an in- 
Figure 6: Point estimates and 95\% confidence intervals for the change in tracking error and return due to imposing the minimum $90 \%$ sustainability target on the frontier of MTE efficient portfolios. ${ }^{\star}$
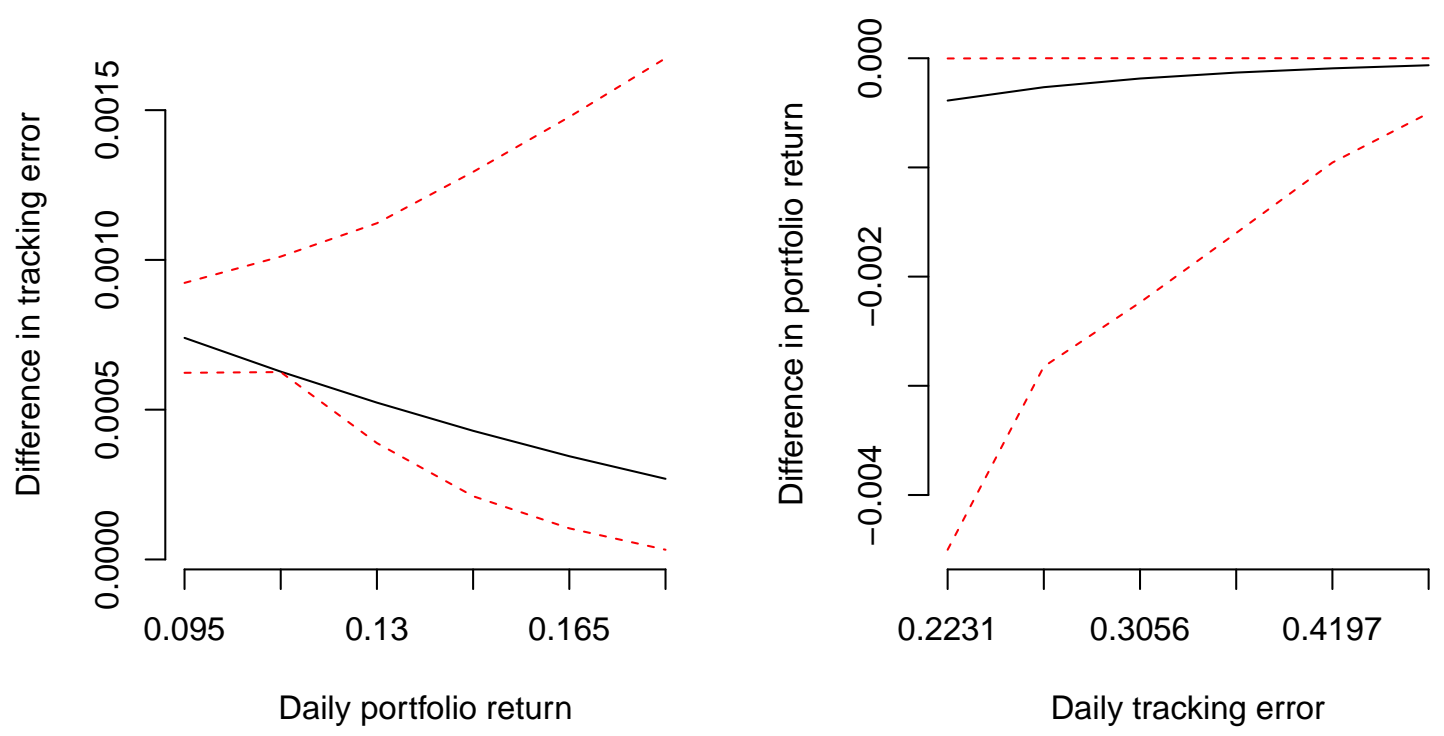

$\star$ Mean return and portfolio variance are for returns expressed in percentage points. 
vestor imposing a constraint on sustainability might not be able to reach certain parts of the unconstrained efficient frontier.

For the US stocks belonging to the MSCI World index in the period 2003-2010, we find that the slope of the relationship between return and sustainability for the MV and MTE efficient portfolios is almost never statistically significantly different from zero. Furthermore, we estimate the increase in variance (for a given target return) and the loss in return (for a given target variance) due to a sustainability constraint and find that the performance loss is economically rather small for MTE efficient portfolios and somewhat larger for MV efficient portfolios.

An interesting topic for future research is to extend our analytical framework to include short selling constraints. Using mean-variance spanning tests, Galema et al. (2008) and Herzel et al. (2011) find that short-selling constraints substantially reduce the impact of restrictions on sustainability. In this paper, we focus on the meantracking error efficient frontier, which we define as the set of portfolios that minimize the tracking error volatility for a given target portfolio return. It would be relevant to extend our analysis to alternative types of efficient frontiers such as the alpha-TrackingError-Volatility frontier discussed in Alexander and Baptista (2010). Another direction for further research is to apply our methodology to study the liquidity of MV and MTE portfolios. Similar to sustainability, liquidity is important for many investors, but ignored in the traditional mean-variance or mean-tracking error framework. As discussed in Lo et al. (2003), portfolio liquidity can be defined as the weighted average of the liquidity of the underlying assets, which then allows to apply the methodology proposed in this paper. 
Acknowledgement: We would like to thank Stephen Hine (EIRIS), David Duchi and Geert Heuninck (KBC Asset Management) and Annabelle Szwed and Jordi Lesaffer (VIGEO) for providing us their database of sustainability scores. Furthermore, we thank Gerda Claeskens, David Veredas and Gunther Wuyts for sharing their comments on the paper. Financial support from the Flemish IWT (Institute for Science and Innovation) and Dutch science foundation is gratefully acknowledged.

\section{References}

Alexander, G. J. and A. M. Baptista (2010). Active portfolio management with benchmarking: A frontier based on alpha. Journal of Banking 6 Finance 34, $2185-$ 2197.

Amenc, N. and V. Le Sourd (2008). Socially responsible investment performance in France. EDHEC Risk and Asset Management Research Centre.

Bauer, R., K. Koedijk, and R. Otten (2005). International evidence on ethical mutual fund performance and investment style. Journal of Banking ES Finance 29, 17511767.

Best, M. and R. Grauer (1990). The efficient set mathematics when mean-variance problems are subject to general linear constraints. Journal of Economics and Business 42, 105-120.

Derwall, J. and K. Koedijk (2009). Socially responsible fixed-income funds. Journal of Business Finance \& Accounting 36, 210-229.

Derwall, J., K. Koedijk, and J. Ter Horst (2011). A tale of values-driven and profitseeking social investors. Journal of Banking 6 Finance 35, 2137-2147.

DiCiccio, T. J. and J. P. Romano (1988). A review of bootstrap confidence intervals. Journal of the Royal Statistical Society Series B 50, 338-354.

Drut, B. (2010). Social responsibility and mean-variance portfolio selection. Working Papers CEB 10-002.RS, ULB.

Galema, R., A. Platinga, and B. Scholtens (2008). Diversification of socially responsible investment portfolios: Testing for mean-variance spanning. Working paper.

Goltz, F. (2009). A long road ahead for portfolio construction: Practitioners' views of an EDHEC survey. EDHEC Risk and Asset Management Research Centre Publication.

Hallerbach, W., H. Ning, A. Soppe, and J. Spronk (2004). A framework for managing a portfolio of socially responsible investments. European Journal of Operational Research 153, 517-529. 
Herzel, S., M. Nicolosi, and C. Starica (2011). The cost of sustainability on optimal portfolio choices. European Journal of Finance 1, 1-17.

Hosseini, M. H. and S. Kaneko (2011). Dynamic sustainability assessment of countries at the macro level: A principal component analysis. Ecological indicators 11, 811823 .

Jorion, P. (2003). Portfolio optimization with tracking-error constraints. Financial Analysts Journal 59, 70-82.

Ledoit, O. and M. Wolf (2003). Improved estimation of the covariance matrix of stock returns with an application to portfolio selection. Journal of Empirical Finance 10, 603-621.

Ledoit, O. and M. Wolf (2004a). Honey, I shrunk the sample covariance matrix. Journal of Portfolio Management 30, 110-119.

Ledoit, O. and M. Wolf (2004b). A well-conditioned estimator for large-dimensional covariance matrices. Journal of Multivariate Analysis 88, 365-411.

Lo, A., C. Petrov, and M. Wierzbicki (2003). It's 11PM - Do you know where your liquidity is? The mean-variance-liquidity frontier. Journal of Investment Management 1, 55-93.

Politis, D. N. and J. P. Romano (1994). The stationary bootstrap. Journal of the American Statistical Association 89, 1303-1313.

Roll, R. (1992). A mean-variance analysis of tracking error. Journal of Portfolio Management 18, 13-22.

Rowe, B. L. Y. (2011). Tawny: various portfolio optimization strategies including random matrix theory and shrinkage estimators. $\mathrm{R}$ package version 1.2.1.

Rudolf, M., H.-J. Wolter, and H. Zimmermann (1999). A linear model for tracking error minimization. Journal of Banking 65 Finance 23, 85 - 103.

Ruiz, E. and L. Pascual (2002). Bootstrapping financial time series. Journal of Economic Surveys 16, 271-300.

Satchell, S. and S. Hwang (2001). Tracking error: Ex ante versus ex post measures. Journal of Asset Management 2, 241-246.

Social Investment Forum (2010). Report on socially responsible investment trends in the United States. http://ussif.org/resources/research/.

Statman, M. and D. Gkushkov (2009). The wages of social responsibility. Financial Analysts Journal 65, 774-800. 


\section{A Appendix}

\section{Derivations mean-variance efficient portfolios.}

The Lagrangian corresponding to the problem in (2.1) is

$$
\mathcal{L}\left(\omega, l_{1}\right)=\omega^{\prime} \mu-\frac{\lambda}{2} \omega^{\prime} \Sigma \omega-l_{1}\left(\omega^{\prime} \iota-1\right) .
$$

The corresponding first order conditions are:

$$
\begin{aligned}
\mu-\lambda \Sigma \omega-l_{1} \iota & =0 \\
\omega^{\prime} \iota & =1 .
\end{aligned}
$$

From (A.1), it follows that

$$
\omega=\frac{1}{\lambda} \Sigma^{-1}\left(\mu-l_{1} \iota\right)
$$

Using (A.2) this gives

$$
\frac{1}{\lambda}\left(\iota^{\prime} \Sigma^{-1} \mu-l_{1} \iota^{\prime} \Sigma^{-1} \iota\right)=1
$$

which can be rewritten as

$$
l_{1}=\frac{-\lambda+\iota^{\prime} \Sigma^{-1} \mu}{\iota^{\prime} \Sigma^{-1} \iota}
$$

Combining (A.3) and (A.4) then gives

$$
\begin{gathered}
\omega_{\mathrm{MV}}(\lambda)=a_{\mathrm{MV}}+\frac{1}{\lambda} b \\
\text { with } a_{\mathrm{MV}}=\frac{\Sigma^{-1} \iota}{\iota^{\prime} \Sigma^{-1} \iota} \text { and } b=\Sigma^{-1}\left(\mu-\iota \frac{\iota^{\prime} \Sigma^{-1} \mu}{\iota^{\prime} \Sigma^{-1} \iota}\right) .
\end{gathered}
$$

\section{Derivations mean-tracking error efficient portfolios.}

Define

$$
\tilde{\Sigma} \equiv\left(\begin{array}{cc}
\sigma_{\mathrm{M}}^{2} & \gamma^{\prime} \\
\gamma & \Sigma
\end{array}\right)
$$

with $\sigma_{\mathrm{M}}^{2}$ the variance of the benchmark return, and $\gamma$ the $(N \times 1)$ vector with the covariances between the benchmark return and the returns of the $N$ assets. Let $\mu_{\mathrm{M}}$ be the mean return of the benchmark. The Lagrangian corresponding to the problem in $(2.2)$ is

$$
\mathcal{L}\left(\tilde{\omega}, l_{1}, l_{2}\right)=\tilde{\omega}^{\prime} \tilde{\mu}-\frac{\lambda}{2} \tilde{\omega}^{\prime} \tilde{\Sigma} \tilde{\omega}-l_{1} \tilde{\omega}^{\prime} \iota-l_{2}\left(\tilde{\omega}^{\prime} j+1\right)
$$

which can be rewritten, using (A.6) and $\tilde{\omega}=(-1, \omega)^{\prime}$, to:

$$
\mathcal{L}\left(\omega, l_{1}\right)=-\mu_{\mathrm{M}}+\omega^{\prime} \mu-\frac{\lambda}{2} \sigma_{\mathrm{M}}^{2}+\lambda \omega^{\prime} \gamma-\frac{\lambda}{2} \omega^{\prime} \Sigma \omega-l_{1}\left(\omega^{\prime} \iota-1\right)
$$


Note that $l_{2}$ disappears in the simplification.

The corresponding first order conditions are:

$$
\begin{aligned}
\mu+\lambda \gamma-l_{1} \iota-\lambda \Sigma \omega & =0 \\
\omega^{\prime} \iota & =1 .
\end{aligned}
$$

Combining (A.7) and (A.8) gives

$$
\frac{1}{\lambda}\left(\iota^{\prime} \Sigma^{-1} \mu+\lambda \iota^{\prime} \Sigma^{-1} \gamma-l_{1} \iota^{\prime} \Sigma^{-1} \iota\right)=1,
$$

which can be rewritten as

$$
l_{1}=\frac{-\lambda+\left(\iota^{\prime} \Sigma^{-1} \mu\right)+\lambda\left(\iota^{\prime} \Sigma^{-1} \gamma\right)}{\iota^{\prime} \Sigma^{-1} \iota} .
$$

Replacing $l_{1}$ in Equation (A.7) with the right-hand-side of (A.9), and then solving for $\omega$ yields

$$
\begin{gathered}
\omega_{\mathrm{MTE}}(\lambda)=a_{\mathrm{MTE}}+\frac{1}{\lambda} b, \\
\text { with } a_{\mathrm{MTE}}=\Sigma^{-1}\left(\frac{\left.\gamma\left(\iota^{\prime} \Sigma^{-1} \iota\right)-\iota \iota \iota^{\prime} \Sigma^{-1} \gamma\right)+\iota}{\iota^{\prime} \Sigma^{-1} \iota}\right),
\end{gathered}
$$

with $b$ as defined in (A.5).

\section{Derivations mean-variance efficient portfolios constrained by sustainabil- ity.}

The Lagrangian corresponding to the problem in (2.13) is

$$
\mathcal{L}\left(\omega, l_{1}, l_{2}\right)=\omega^{\prime} \mu-\frac{\lambda}{2} \omega^{\prime} \Sigma \omega-l_{1}\left(\omega^{\prime} \iota-1\right)-l_{2}\left(\omega^{\prime} \phi-\phi_{0}\right) .
$$

The corresponding first order conditions are:

$$
\begin{aligned}
\mu-\lambda \Sigma \omega-l_{1} \iota-l_{2} \phi & =0, \\
\omega^{\prime} \iota & =1, \\
\omega^{\prime} \phi & =\phi_{0} .
\end{aligned}
$$

From (A.12), it follows that

$$
\omega=\frac{\Sigma^{-1}}{\lambda}\left(\mu-l_{1} \iota-l_{2} \phi\right) .
$$

Now

$$
\iota^{\prime} \omega=\frac{1}{\lambda}\left(\iota^{\prime} \Sigma^{-1} \mu-l_{1} \iota^{\prime} \Sigma^{-1} \iota-l_{2} \iota^{\prime} \Sigma^{-1} \phi\right)=1,
$$


which can be rewritten as

$$
l_{1}=\frac{-\lambda+\iota^{\prime} \Sigma^{-1} \mu-l_{2} \iota^{\prime} \Sigma^{-1} \phi}{\iota^{\prime} \Sigma^{-1} \iota} .
$$

Combining (A.15) and (A.16) then yields

$$
\omega_{\mathrm{MV}}(\lambda)=a_{\mathrm{MV}}+\frac{b}{\lambda}+\frac{c l_{2}}{\lambda}
$$

with $a_{\mathrm{MV}}$ and $b$ as defined in (A.5) and

$$
c=\Sigma^{-1}\left\{\left(\frac{\iota^{\prime} \Sigma^{-1} \phi}{\iota^{\prime} \Sigma^{-1} \iota}\right) \iota-\phi\right\} .
$$

From (A.14) we know that in case the sustainability constraint is binding

$$
\omega_{\mathrm{MV}}^{\prime}(\lambda) \phi=a_{\mathrm{MV}}^{\prime} \phi+\frac{b^{\prime} \phi}{\lambda}+l_{2} \frac{c^{\prime} \phi}{\lambda}=\phi_{0},
$$

which can be rewritten to find $l_{2}$ as follows

$$
l_{2}=\frac{\lambda \phi_{0}-b^{\prime} \phi-\lambda a_{\mathrm{MV}}^{\prime} \phi}{c^{\prime} \phi} .
$$

\section{Derivations mean-tracking error efficient portfolios constrained by sus- tainability.}

The Lagrangian corresponding to the problem in (2.16) is

$$
\mathcal{L}\left(\omega, l_{1}, l_{2}\right)=-\mu_{M}+\omega^{\prime} \mu-\frac{\lambda}{2} \sigma_{M}^{2}-\frac{\lambda}{2} \omega^{\prime} \Sigma \omega+\lambda \omega^{\prime} \gamma-l_{1}\left(\omega^{\prime} \iota-1\right)-l_{2}\left(\omega^{\prime} \phi-\phi_{0}\right) .
$$

The corresponding first order conditions are:

$$
\begin{aligned}
\mu-\lambda \Sigma \omega+\lambda \gamma-l_{1} \iota-l_{2} \phi & =0, \\
\omega^{\prime} \iota & =1, \\
\omega^{\prime} \phi & =\phi_{0} .
\end{aligned}
$$

From (A.18), it follows that

$$
\omega=\frac{\Sigma^{-1}}{\lambda}\left(\mu-l_{1} \iota-l_{2} \phi\right)+\Sigma^{-1} \gamma .
$$

Now, from (A.19) we know that

$$
\iota^{\prime} \omega=\frac{1}{\lambda}\left(\iota^{\prime} \Sigma^{-1} \mu-l_{1} \iota^{\prime} \Sigma^{-1} \iota-l_{2} \iota^{\prime} \Sigma^{-1} \phi\right)+\iota^{\prime} \Sigma^{-1} \gamma=1,
$$


which can be rewritten as

$$
l_{1}=\frac{-\lambda+\iota^{\prime} \Sigma^{-1} \mu+\lambda \iota^{\prime} \Sigma^{-1} \gamma-l_{2} \iota^{\prime} \Sigma^{-1} \phi}{\iota^{\prime} \Sigma^{-1} \iota} .
$$

Combining (A.21) and (A.22) then yields

$$
\omega_{\mathrm{MTE}}\left(\lambda, \phi_{0}\right)=a_{\mathrm{MTE}}+\frac{b}{\lambda}+\frac{c}{\lambda} l_{2},
$$

with $a_{\mathrm{MTE}}$ given in (A.11), $b$ as defined in (A.5), and $c$ as defined in (A.17). From (A.20) we know that in case the sustainability constraint is binding

$$
\omega_{\mathrm{MTE}}^{\prime}\left(\lambda, \phi_{0}\right) \phi=a_{\mathrm{MTE}}^{\prime} \phi+\frac{b^{\prime} \phi}{\lambda}+l_{2} \frac{c^{\prime} \phi}{\lambda}=\phi_{0},
$$

which can be rewritten to find $l_{2}$ as

$$
l_{2}=\frac{\lambda \phi_{0}-b^{\prime} \phi-\lambda a_{\mathrm{MTE}}^{\prime} \phi}{c^{\prime} \phi} .
$$

\section{The Ledoit-Wolf shrinkage procedure to covariance estimation.}

When the number of assets $(N)$ is large compared to the number of time series observations $(T)$, Ledoit and Wolf $(2003,2004 \mathrm{~b})$ recommend to estimate the covariance matrix $\Sigma$ in the following way:

$$
\hat{\Sigma}=\hat{\delta}^{*} F+\left(1-\hat{\delta}^{*}\right) S
$$

with $\hat{\delta}^{*}$ the estimated optimal shrinkage intensity, $S$ the sample covariance matrix, and $F$ the shrinkage target. The shrinkage target is based on the constant correlation model, which assumes that all (pairwise) correlations are identical. The $(i, j)$ th element of $F$ is given by $f_{i j}=\bar{r} \sqrt{s_{i i} s_{j j}}$, with $\bar{r}$ the average estimated pairwise correlation and $s_{i i}$ the $(i, i)$ th element of $S$, i.e. the estimated variance of the $i$-th asset. Denote by $\phi_{i j}=\bar{\varrho} \sqrt{\sigma_{i i} \sigma_{j j}}$, with $\bar{\varrho}$ the average true population correlation and $\sigma_{i i}$ the $(i, i)$ th element of $\Sigma$, i.e. the true variance of the $i$-th asset. The optimal shrinkage intensity $\delta^{*}$ needs to be selected. Given that $S$ is consistent and $F$ is not, with $N$ fixed and $T$ going to infinity, the optimal shrinkage intensity goes to zero asymptotically. Define the Frobenius norm of an $(N \times N)$ matrix $Z$ with elements $z_{i j}$ by $\|Z\|^{2}=\sum_{i=1}^{N} \sum_{j=1}^{N} z_{i j}^{2}$. The goal is then to find the shrinkage intensity $\delta^{*}$ which minimizes the expected value of the loss function $L(\delta)=\|\delta F+(1-\delta) S-\Sigma\|^{2}$. We estimate the shrinkage intensity $\delta^{*}$ by

$$
\hat{\delta}^{*}=\max \{0, \min \{\hat{\kappa}, 1\}\}, \text { with } \hat{\kappa}=\frac{\hat{\pi}-\hat{\rho}}{\hat{\gamma}} .
$$

Here, $\hat{\pi}$ is an estimator for $\pi$, with $\pi$ the sum of the asymptotic variances of the entries of the sample covariance matrix scaled by $\sqrt{T}: \pi=\sum_{i=1}^{N} \sum_{j=1}^{N} \operatorname{Asy} \operatorname{Var}\left(\sqrt{T} s_{i j}\right)$. Further, in Equation (A.25) $\hat{\rho}$ is the estimator for $\rho$, which denotes the sum of the 
asymptotic covariances of the entries of the shrinkage target with the entries of the sample covariance matrix scaled by $\sqrt{T}: \rho=\sum_{i=1}^{N} \sum_{j=1}^{N} \operatorname{AsyCov}\left(\sqrt{T} f_{i j}, \sqrt{T} s_{i j}\right)$. Finally, $\hat{\gamma}$ is an estimator for $\gamma$ which measures the misspecification of the (population) shrinkage target: $\gamma=\sum_{i=1}^{N} \sum_{j=1}^{N}\left(\phi_{i j}-\sigma_{i j}\right)^{2}$. More details can be found in Ledoit and Wolf (2004a). ${ }^{2}$

\section{The bias-corrected accelerated percentile method.}

We explain briefly the procedure here and refer to DiCiccio and Romano (1988) for more details. Suppose we are interested in the confidence regions around the estimate $\hat{\theta}$ of the unknown parameter $\theta$. Denote by $\hat{\theta}_{(1)}^{*} \leq \hat{\theta}_{(2)}^{*} \leq \ldots \leq \hat{\theta}_{(B)}^{*}$ the set of parameter estimates based on the $B$ bootstrapped samples. Calculate

$$
z_{0}=\Phi^{-1}\left(\left(\frac{1}{B}\right) \sum_{b=1}^{B} I\left(\hat{\theta}_{(b)}^{*} \leq \hat{\theta}\right)\right)
$$

with $\Phi^{-1}$ the inverse of the cumulative distribution function of the standard normal distribution, and $I$ the indicator function. Then calculate the two bias-corrected quantiles as follows: $v_{1}=\Phi\left(z_{0}+\left(z_{0}+z_{\alpha / 2}\right) /\left(1-a\left(z_{0}+a z_{\alpha / 2}\right)\right)\right)$ and $v_{2}=\Phi\left(z_{0}+\left(z_{0}+\right.\right.$ $\left.\left.z_{1-\alpha / 2}\right) /\left(1-a\left(z_{0}+a z_{1-\alpha / 2}\right)\right)\right)$, with $z_{\alpha}=\Phi^{-1}(\alpha)$ and $a$ the acceleration factor. For the estimation of $a$, the full data set is subdivided in blocks with stochastic lengths as described above. Denote by $R=\left\{r_{(1)}, \ldots, r_{(D)}\right\}$ the full data set, with $r_{(j)}$ the return vector at day $j$ and $D$ the total number of days in the data set. The acceleration $a$ is estimated by

$$
\hat{a}=\frac{1}{6} \frac{\sum_{i=1}^{D} U_{i}^{3}}{\left(\sum_{i=1}^{D} U_{i}^{2}\right)^{3 / 2}},
$$

with $U_{i}=(D-z)\left(\hat{\theta}-\hat{\theta}_{(i)}\right)$, where $\hat{\theta}_{(i)}$ is the estimate of $\theta$ based on a reduced data set $R_{(i)}$. The reduced data set used for the estimation of $\hat{\theta}_{(i)}$ is given by the full data set excluding return vectors which are in the same block as $r_{(i)}$, i.e. $R_{(i)}=$ $\left\{r_{(1)}, \ldots, r_{(s-1)}, r_{(e+1)}, \ldots, r_{(D)}\right\}$ with $s$ and $e$ indicating the start and end of the block containing $r_{(i)}$ respectively, and $z=e-s+1$ the length of that block. Finally, the $100(1-\alpha) \%$ confidence region is then given by $\left[\hat{\theta}_{\left((B+1) v_{1}\right)}^{*}, \hat{\theta}_{\left((B+1) v_{2}\right)}^{*}\right]$.

\footnotetext{
${ }^{2}$ The estimation of $\delta^{*}$ is performed by the function shrinkage.intensity from the R-package tawny (Rowe, 2011).
} 